\title{
Efficacy of Generic Atorvastatin in a Real-World Setting
}

This article was published in the following Dove Press journal:

Clinical Pharmacology: Advances and Applications

\author{
Panisa Manasirisuk' \\ Nanthaphan Chainirun ${ }^{1,2}$ \\ Somsak Tiamkao (iD) 2,3 \\ Sunee Lertsinudom ${ }^{4}$ \\ Kutcharin Phunikhom ${ }^{5}$ \\ Bundit Sawunyavisuth ${ }^{6}$ \\ Kittisak Sawanyawisuth iD ${ }^{3}$ \\ 'Department of Pharmacy Service, \\ Srinagarind Hospital, Faculty of Medicine, \\ Khon Kaen University, Khon Kaen, \\ Thailand; ${ }^{2}$ Integrated Epilepsy Research \\ Group, Khon Kaen University, Khon \\ Kaen, Thailand; ${ }^{3}$ Department of \\ Medicine, Faculty of Medicine, Khon \\ Kaen University, Khon Kaen, Thailand; \\ ${ }^{4}$ Division of Pharmaceutical Care, Faculty \\ of Pharmaceutical Sciences, Khon Kaen \\ University, Khon Kaen, Thailand; \\ ${ }^{5}$ Department of Pharmacology, Faculty of \\ Medicine, Khon Kaen University, Khon \\ Kaen, Thailand; ${ }^{6}$ Department of \\ Marketing, Faculty of Business \\ Administration and Accountancy, Khon \\ Kaen University, Khon Kaen, Thailand
}

Correspondence: Nanthaphan Chainirun Department of Pharmacy Service, Srinagarind Hospital, Faculty of Medicine, Khon Kaen University, Khon Kaen, 40002, Thailand

Email kanokwanchainirun@yahoo.com

Kittisak Sawanyawisuth

Department of Medicine, Faculty of

Medicine, Khon Kaen University, Khon

Kaen, 40002, Thailand

Tel +66-43-363664

Fax +66-43-348399

Email kittisak@kku.ac.th
Background: The ability of statins to reduce LDL-c plays an important role in both primary and secondary prevention of atherosclerotic cardiovascular diseases. Such treatment can often be costly, but using generic atorvastatin may reduce cost by up to US\$2635. In addition, a previous 8-week study found that it exhibited comparable efficacy to the brandname medication. This study aimed to evaluate the efficacy of generic atorvastatin over a longer period of six months in a real-world setting.

Methods: This was a retrospective cohort study in adult patients who had received brandname atorvastatin for at least three months and then had switched to generic atorvastatin for at least six months. Lipid and safety profiles were evaluated at six months after switching. Adjusted analyses for age, sex, co-morbid disease, dosage, and indications for statin therapy were also performed.

Results: During the study period, there were 488 patients who met the study criteria. The mean (SD) age of the patients was 60.97 (12.26) years, and $48.36 \%$ were male (236 patients). At six months, average total cholesterol, HDL-c, and LDL-c were all lower, from 174.43 to $166.15 \mathrm{mg} / \mathrm{dL}$, from 51.64 to $49.51 \mathrm{mg} / \mathrm{dL}$, and from 110.08 to $100.78 \mathrm{mg} / \mathrm{dL}$ ( $\mathrm{p}<0.001$ ), respectively. There were no significant differences in terms of any other laboratory test results. LDL-c exhibited the highest significant reduction at $9.30 \mathrm{mg} / \mathrm{dL}$. Stratified analyses by age, sex, co-morbid disease, dose, and indications for statin therapy revealed similar decreases in HDL-c and LDL-c as in the study population as a whole.

Conclusion: Generic atorvastatin resulted in significantly lower LDL-c than name-brand atorvastatin but less of an increase in HDL-c.

Keywords: low-density lipoprotein cholesterol, high-density lipoprotein cholesterol, HMG CoA reductase inhibitors

\section{Introduction}

Dyslipidemia is a contributing factor to major cardiovascular diseases. In 2013, there were approximately 17 million deaths globally from cardiovascular diseases. ${ }^{1}$ Approximately half of patients with established cardiovascular diseases have accompanied dyslipidemia, ${ }^{2}$ the prevalence of which is up to $85 \%$ higher in patients with premature coronary heart disease. ${ }^{3,4}$ As low-density lipoprotein cholesterol (LDL-c) is the main cause of atherosclerosis, the ability of HMG CoA reductase inhibitors (statins) to reduce LDL-c plays an important role in both primary and secondary prevention of atherosclerotic cardiovascular diseases.

Despite the benefits of statin therapy for patients with atherosclerotic cardiovascular diseases, this type of treatment can be expensive. The use of generic statins may help to mitigate these costs. A previous study found the medical costs incurred by patients 
using brand-name medications to be three times higher than in those using generic drugs. ${ }^{5}$ In addition, the average income in the former group was 10 times higher than that in the latter. ${ }^{5}$ Although treatment with generic atorvastatin could save patients an estimated US\$2635, ${ }^{6}$ data are limited with regard to its clinical efficacy. However, a 2013 study from Korea found the efficacy of generic atorvastatin to be on par with that of the name-brand version over an 8-week period. ${ }^{7}$ Several other studies found generic atorvastatin to be effective over periods ranging from 8-12 weeks, ${ }^{8,9}$ the largest of which had a sample size of 266 patients. $^{10,11}$ This study aimed to evaluate the efficacy of generic atorvastatin over a longer period (six months) and with a larger sample size in a real-world setting.

\section{Methods}

This was a retrospective cohort study conducted at Srinagarind Hospital, a 1000-bed University Hospital in Khon Kaen, Thailand. Adult patients who had received brand-name atorvastatin for at least three months and were then put on a generic version for at least six months at the same dosage. Those who had taken other lipid-lowering medications or medications that affect lipid levels, who had no follow-up lipid profile data, or who had been given differing doses of atorvastatin during the treatment period were excluded. Those taking gemfibrozil, fenofibrate, ezetimibe, cholestyramine, prednisolone, dexamethasone, hydrocortisone, or fludrocortisone as concomitant medications were also excluded. The brand-name atorvastatin used was Xarator (Pfizer Pharmaceuticals, Peurto Rico), while the generic was Atorvastatin Sandoz (Lek Pharmaceuticals, Slovenia). The generic atorvastatin had a bioequivalence of over $90 \%$ to the brand-name atorvastatin to be approved by the Thai Food Drug Administration. The prescriptions in this study were filled by the patients. The study period was between October 2016 and March 2018.

Data of eligible patients were retrieved from the hospital's electronic database. Clinical data were reviewed and recorded including age, sex, education level, insurance, co-morbid diseases, atorvastatin dosage, body mass index, lipid profiles (total cholesterol, triglyceride, high-density lipoprotein cholesterol [HDL-c], and LDL-c), serum blood urea nitrogen, serum creatinine, estimated glomerular filtration rate, serum alanine aminotransferase (ALT), serum aspartate aminotransferase (AST), and serum creatinine kinase $(\mathrm{CK})$. Laboratory results at baseline or prior to treatment with generic atorvastatin and six months after initiation of treatment were recorded and compared. The study protocol was approved by the ethic committee in human research, Khon Kaen University, Khon Kaen, Thailand and complied with the Declaration of Helsinki. An informed consent was not required by the ethic committee to retrospective data collection and patients data are confidential.

\section{Statistical Analyses}

A paired $t$-test was used to compute differences between the two time points. Data were presented as means (SD), and mean differences between baseline and at six months with their 95\% confidence intervals (CIs). Lipid profiles were adjusted for age, sex, common co-morbid disease, dosage, and indications for statin therapy. Atorvastatin treatment was considered to be secondary prevention in patients who had suffered from coronary artery disease or stroke. Differences with $\mathrm{p}$ values less than 0.05 were considered statistically significant. Statistical analyses were performed using STATA (College Station, Texas, USA).

\section{Results}

During the study period, there were 1044 patients who had switched treatment from brand-name atorvastatin to the generic version. Of those, 556 were excluded due to concurrent treatment with lipid-lowering agents (240 patients), lack of follow-up lipid profile data (187 patients), and differing atorvastatin dosages (129 patients), as shown in Figure 1. In total, there were 488 patients who met the study criteria. The mean (SD) patient age was 60.97 (12.26) years and $48.36 \%$ were male (236 patients). Most commonly, patients had basic health insurance (206 patients; 42.21\%), and $17.21 \%$ were unemployed (Table 1). Hypertension was the most common co-morbid disease (272 patients; 55.74\%). Atorvastatin treatment was administered as secondary prevention in 93 patients $(19.06 \%)$. The two most common dosages were 20 and $40 \mathrm{mg} /$ day (42.42\% and 46.13\%).

Three factors differed significantly after six months of treatment with the generic atorvastatin including total cholesterol level, HDL-c level, and LDL-c level ( $\mathrm{p}$ value < 0.001 ), as shown in Table 1. At six months, total cholesterol, HDL-c and LDL-c had decreased from 174.43 to $166.15 \mathrm{mg} / \mathrm{dL}$, from 51.64 to $49.51 \mathrm{mg} / \mathrm{dL}$, and from 110.08 to $100.78 \mathrm{mg} / \mathrm{dL}$, respectively. No other laboratory test results differed significantly. The highest significant reduction was in LDL-c, at $9.30 \mathrm{mg} / \mathrm{dL}$ (Table 2). Stratified analyses by age, sex, co-morbid disease, dosage, and indications for statin therapy showed decreases in HDL-c and LDL-c similar to those of the study population as a whole (Tables 3-7). The largest LDL-c reduction was found in those under 60 years of age at $-11.90 \mathrm{mg} / \mathrm{dL}$. 


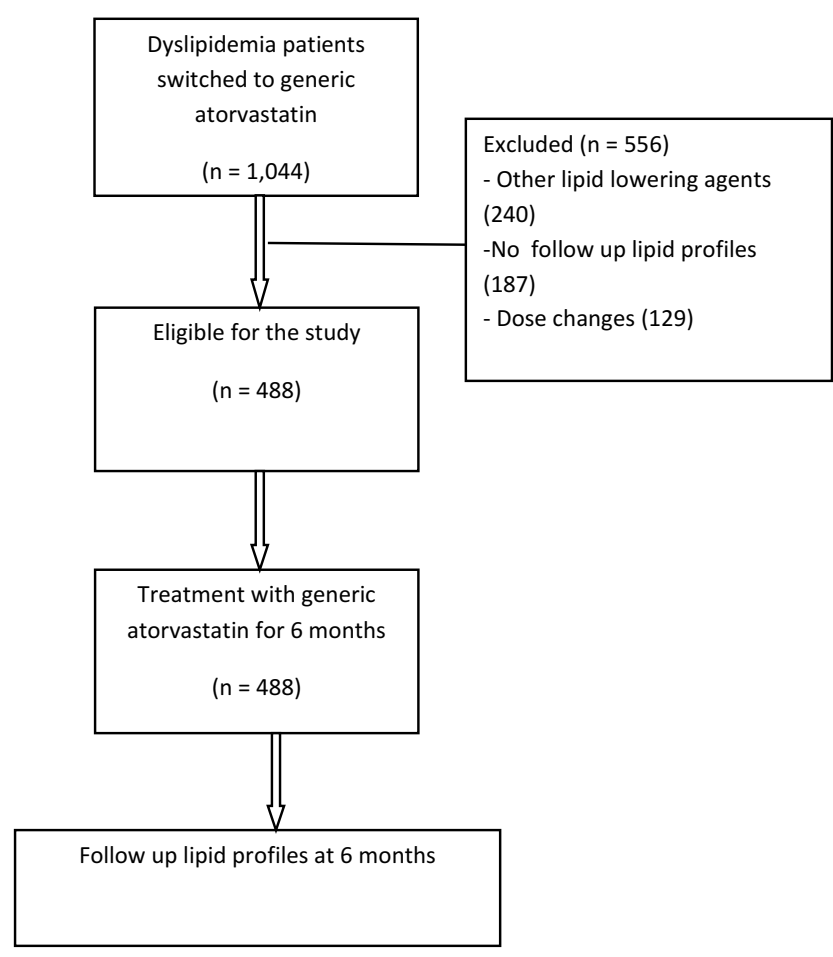

Figure I Study flow.

\section{Discussion}

This real-world, long-term evaluation of generic atorvastatin revealed significant reductions in LDL-c compared with brand-name atorvastatin. Several studies have demonstrated atorvastatin's ability to significantly reduce LDL-c levels. ${ }^{7-12}$ One study, for example, showed that brand-name atorvastatin was able to lower LDL-c by $42 \%$ after 8 weeks of treatment. ${ }^{12}$ In this study, there was no such reduction from pre-treatment, but treatment with the generic formulation resulted in significant reductions compared with the brand-name medication. This differed from another previous study, which found no significant differences between these two regimens (LDL difference of -2.32 to 5.24$){ }^{7}$ While there have also been other studies showing no significant differences between brand-name and generic atorvastatin, ${ }^{8-11}$ we found in this study that switching to generic atorvastatin resulted in an average LDL reduction of $9.30 \mathrm{mg} / \mathrm{dL}$, with a $95 \%$ CI of $12.99 \mathrm{mg} / \mathrm{dL}$. These contrasting findings may be explained by the larger sample size in this study, the longer study period of 6 months, and/or differences in the type of generic atorvastatin used. ${ }^{7,8,11}$ Generic atorvastatin may also result in LDL-c reductions similar to those from rosuvastatin ( $\mathrm{p} \mathrm{0.91).}{ }^{1}$ Though atorvastatin is reported to increase HDL-c by $6-7 \%,{ }^{14}$ our generic atorvastatin had
Table I Clinical Parameters of Dyslipidemia Patients Who Switched Treatment from Brand-Name Atorvastatin to Generic Atorvastatin

\begin{tabular}{|c|c|}
\hline Factors & Mean (SD) or Number (\%) \\
\hline Age & $60.97(12.26)$ \\
\hline Male sex & $236(48.36)$ \\
\hline \multicolumn{2}{|l|}{ Education } \\
\hline Primary school & $7(1.43)$ \\
\hline Secondary school & $4(0.82)$ \\
\hline College & $3(0.6 \mathrm{I})$ \\
\hline Unknown & $475(97.34)$ \\
\hline \multicolumn{2}{|l|}{ Insurance } \\
\hline Basic & $206(42.21)$ \\
\hline Social security & 95 (19.47) \\
\hline Government & I6I (32.99) \\
\hline Self-pay & $25(5.12)$ \\
\hline \multicolumn{2}{|l|}{ Occupation } \\
\hline Government officer & II (2.25) \\
\hline Private company & $45(9.22)$ \\
\hline Farmer & $31(6.35)$ \\
\hline Unemployed & $84(17.2 I)$ \\
\hline Unknown & $313(64.14)$ \\
\hline \multicolumn{2}{|l|}{ Co-morbid disease } \\
\hline Hypertension & $272(55.74)$ \\
\hline Atrial fibrillation & $17(3.48)$ \\
\hline Diabetes & $222(45.49)$ \\
\hline Chronic kidney disease & $56(11.48)$ \\
\hline Heart failure & $16(3.28)$ \\
\hline Coronary artery disease & $62(12.70)$ \\
\hline Stroke & $31(6.35)$ \\
\hline \multicolumn{2}{|l|}{ Dose of atorvastatin, $\mathrm{mg}$} \\
\hline 10 & $3(0.61)$ \\
\hline 20 & $207(42.42)$ \\
\hline 40 & $270(46.13)$ \\
\hline 60 & $7(1.43)$ \\
\hline 80 & I $(0.20)$ \\
\hline
\end{tabular}

less of an effect on HDL-c than the brand-name medication, with lower average HDL-c levels than at baseline $(-2.13 \mathrm{mg} / \mathrm{dL})$. As a result of LDL-c and HDL-c reduction, total cholesterol was also significantly lower than at baseline (-8.28). Results adjusted for age, sex, co-morbid disease, dosage, and indications for statin therapy were similar to those of the overall population.

Regarding safety profiles, only ALT levels were slightly higher (by $4.37 \mathrm{U} / \mathrm{L}$ ) in the generic versus the name-brand group. However, theses increases were not clinically significant, as the mean value was lower than 
Table 2 Clinical Parameters of Dyslipidemia Patients Before Switching Treatment from Brand-Name to Generic Atorvastatin and 6 Months After

\begin{tabular}{|l|l|l|l|l|l|}
\hline Factors & Baseline & 6th Month & Mean Differences & 95\% CI & P value \\
\hline BMI & $26.57(5.36)$ & $26.48(5.33)$ & -0.09 & $-0.25,0.07$ & 0.285 \\
\hline Chol & $174.43(49.58)$ & $166.15(44.25)$ & -8.28 & $-12.38,-4.19$ & $-5.48,8.19$ \\
\hline Tg & $144.20(69.71)$ & $145.55(77.50)$ & 1.35 & $-3.08,-1.17$ & 0.696 \\
\hline HDL & $51.64(13.43)$ & $49.5 I(13.04)$ & -2.13 & $-12.99,-5.59$ & $<0.001$ \\
\hline LDL & $110.08(44.72)$ & $100.78(43.03)$ & -9.30 & $-0.49,1.50$ & $-0.02,0.05$ \\
\hline BUN & $17.32(10.57)$ & $17.82(12.32)$ & 0.50 & $-0.93,0.74$ & 0.323 \\
\hline Cr & $1.22(1.14)$ & $1.23(I .12)$ & 0.01 & $-7.29,5.74$ & 0.496 \\
\hline eGFR & $72.93(26.44)$ & $72.84(26.97)$ & -0.09 & $-2.34,11.10$ \\
\hline AST & $30.85(28.27)$ & $30.07(21.08)$ & -0.78 & $-79.70,20.78$ & 0.828 \\
\hline ALT & $30.30(17.00)$ & $34.67(37.73)$ & 4.37 & -29.46 & $0.84 I$ \\
\hline CK & $235.30(107.31)$ & $205.84(90.82)$ & 0.225 \\
\hline
\end{tabular}

Abbreviations: $\mathrm{Cl}$, confidence interval; BMI, body mass index $\left(\mathrm{kg} / \mathrm{m}^{2}\right)$; Chol, cholesterol $(\mathrm{mg} / \mathrm{dL})$; $\mathrm{Tg}$, triglyceride $(\mathrm{mg} / \mathrm{dL})$; HDL, high-density lipoprotein-cholesterol (mg/ $\mathrm{dL})$; LDL, low-density lipoprotein-cholesterol (mg/dL); BUN, blood urea nitrogen $(\mathrm{mg} / \mathrm{dL})$; Cr, creatinine $(\mathrm{mg} / \mathrm{dL})$; eGFR, estimated glomerular filtration rate (mL/min/ $\left.1.73 \mathrm{~m}^{2}\right)$; ALT, alanine aminotransferase (U/L); AST, aspartate aminotransferase (U/L); CK, creatinine kinase $(\mathrm{U} / \mathrm{L})$.

Table 3 Lipid Parameters of Dyslipidemia Patients Before Switching Treatment from Brand-Name to Generic Atorvastatin and 6 Months After Stratified by Age

\begin{tabular}{|c|c|c|c|c|c|}
\hline Factors & Baseline & 6th Month & Mean Differences & $95 \% \mathrm{Cl}$ & p value \\
\hline \multicolumn{6}{|l|}{ Age } \\
\hline \multicolumn{6}{|c|}{$<60(n=216)$} \\
\hline Chol & 180.85 (54.92) & $170.04(46.24)$ & -9.58 & $-15.38,-3.78$ & 0.001 \\
\hline $\mathrm{Tg}$ & | 49.82 (75.60) & I57.I7 (91.39) & 4.10 & $-7.1 I, \mid 15.31$ & $0.47 I$ \\
\hline HDL & $52.08(\mid 3.7 I)$ & $49.21(12.75)$ & -2.39 & $-3.74,-1.04$ & $<0.001$ \\
\hline LDL & II 5.66 (49.73) & $103.76(40.51)$ & -11.90 & $-16.76,-7.04$ & $<0.001$ \\
\hline \multicolumn{6}{|c|}{$\geq 60(n=272)$} \\
\hline Chol & $169.43(43.30)$ & $162.00(40.07)$ & -7.18 & $-12.96,-1.39$ & 0.015 \\
\hline $\mathrm{Tg}$ & $137.95(63.12)$ & $137.44(63.86)$ & -1.01 & $-9.37,7.33$ & 0.810 \\
\hline HDL & $51.38(13.47)$ & $49.24(13.21)$ & -1.89 & $-3.24,-0.54$ & 0.006 \\
\hline LDL & 105.65 (39.85) & $98.42(44.87)$ & -7.23 & $-|2.64,-| .8 \mid$ & 0.009 \\
\hline
\end{tabular}

Abbreviations: $\mathrm{Cl}$, confidence interval; Chol, cholesterol (mg/dL); $\mathrm{Tg}$, triglyceride $(\mathrm{mg} / \mathrm{dL})$; $\mathrm{HDL}$, high-density lipoprotein-cholesterol (mg/dL); $\mathrm{LDL}$, low-density lipoprotein-cholesterol (mg/dL).

$40 \mathrm{U} / \mathrm{L}$. Both brand-name and generic atorvastatin had good safety profiles. ${ }^{7,12}$ Atorvastatin may cause liver toxicity 1.4 times compared with simvastatin $(0.32 \%)$ of cases, but it can be severe, particularly with high doses. ${ }^{15}$ A previous report found that atorvastatin at a dosage of
40-80 mg was 7.3 times more likely to cause severe hepatotoxicity (AST or ALT $>400 \mathrm{U} / \mathrm{L}$ ) than low-dose simvastatin. ${ }^{15}$ Another study from Japan reported that one out of 26 patients $(3.84 \%)$ receiving atorvastatin at $10 \mathrm{mg}$ had ALT 2-3 times the upper normal limit. 
Table 4 Lipid Parameters of Dyslipidemia Patients Before Switching Treatment from Brand-Name to Generic Atorvastatin and 6 Months After Stratified by Sex

\begin{tabular}{|c|c|c|c|c|c|}
\hline Factors & Baseline & 6th Month & Mean Differences & $95 \% \mathrm{Cl}$ & p value \\
\hline \multicolumn{6}{|l|}{ Sex } \\
\hline \multicolumn{6}{|c|}{ Female $(n=252)$} \\
\hline Chol & I 78.30 (47.79) & 171.17 (45.08) & -7.12 & $-12.88,-1.37$ & 0.015 \\
\hline $\mathrm{Tg}$ & I 40.98 (65.84) & $|4| .07$ (7I.80) & 0.08 & $-9.36,9.53$ & 0.985 \\
\hline HDL & 53.74 (13.92) & $52.05(13.05)$ & -1.68 & $-3.07,-0.30$ & 0.017 \\
\hline LDL & III.78 (42.38) & $102.30(36.98)$ & -9.48 & $-13.89,-5.07$ & $<0.001$ \\
\hline \multicolumn{6}{|c|}{ Male $(n=236)$} \\
\hline Chol & $\mid 70.76(5 \mid .08)$ & $161.37(43.03)$ & -9.38 & $-15.24,-3.53$ & 0.001 \\
\hline $\mathrm{Tg}$ & I47.53 (73.5I) & $|50.2|$ (82.9|) & 2.67 & $-7.31,12.65$ & 0.598 \\
\hline HDL & 49.52 (12.59) & $46.94(12.56)$ & -2.58 & $-3.90,-1.25$ & $<0.001$ \\
\hline LDL & $108.27(47.11)$ & 99.16 (48.69) & -9.10 & $-15.16,-3.03$ & 0.003 \\
\hline
\end{tabular}

Abbreviations: $\mathrm{Cl}$, confidence interval; $\mathrm{Chol}$, cholesterol $(\mathrm{mg} / \mathrm{dL}) ; \mathrm{Tg}$, triglyceride $(\mathrm{mg} / \mathrm{dL}) ; \mathrm{HDL}$, high-density lipoprotein-cholesterol $(\mathrm{mg} / \mathrm{dL}) ; \mathrm{LDL}, \mathrm{low}-\mathrm{density}$ lipoprotein-cholesterol (mg/dL).

Table 5 Lipid Parameters of Dyslipidemia Patients Before Switching Treatment from Brand-Name to Generic Atorvastatin and 6 Months After Stratified by Co-Morbid Disease

\begin{tabular}{|c|c|c|c|c|c|}
\hline Factors & Baseline & 6th Month & Mean Differences & $95 \% \mathrm{Cl}$ & p value \\
\hline \multicolumn{6}{|c|}{ Hypertension } \\
\hline \multicolumn{6}{|c|}{ No $(n=216)$} \\
\hline Chol & 174.82 (49.83) & $165.58(46.57)$ & -9.23 & $-14.97,-3.49$ & 0.001 \\
\hline $\mathrm{Tg}$ & | 42.48 (69.98) & $|4| .24$ (76.05) & -1.24 & $-10.98,8.50$ & 0.801 \\
\hline HDL & $51.84(13.74)$ & 49.57 (13.29) & -2.27 & $-3.58,-0.95$ & $<0.001$ \\
\hline LDL & III.I4 (46.48) & $103.88(52.05)$ & -7.25 & $-13.44,-1.06$ & 0.021 \\
\hline \multicolumn{6}{|c|}{ Yes $(n=272)$} \\
\hline Chol & $174.12(49.50)$ & $166.62(42.32)$ & -7.49 & $-13.31,-1.67$ & 0.011 \\
\hline $\mathrm{Tg}$ & $145.62(69.61)$ & 149.12 (78.66) & 3.50 & $-6.10,13.10$ & 0.473 \\
\hline HDL & $51.48(13.20)$ & 49.47 (12.87) & -2.01 & $-3.38,-0.63$ & 0.004 \\
\hline LDL & $109.24(43.34)$ & $98.32(34.12)$ & -10.91 & $-15.40,-6.43$ & $<0.001$ \\
\hline
\end{tabular}

Abbreviations: $\mathrm{Cl}$, confidence interval; Chol, cholesterol (mg/dL); $\mathrm{Tg}$, triglyceride (mg/dL); HDL, high-density lipoprotein-cholesterol (mg/dL); LDL, low-density lipoprotein-cholesterol (mg/dL).

There were some limitations to this study. First, some factors associated with atherosclerotic cardiovascular diseases were not studied. ${ }^{16-18}$ In addition, lifestyle modification was not evaluated, as this was a retrospective study and because it may be difficult to monitor patients' lifestyles in a real-world setting. We assumed that the patients as a whole had not made significant lifestyle modifications, as any changes would vary from patient to patient. We also assumed that the patients exhibited good compliance as they had refilled their prescriptions regularly and had not missed any follow-up visits for at least nine months. Some data were limited or missing including education level, socioeconomic status, and baseline lipid values. Finally, as this was a retrospective, observational 
Table 6 Lipid Parameters of Dyslipidemia Patients Before Switching Treatment from Brand-Name to Generic Atorvastatin and 6 Months After Stratified by Dose

\begin{tabular}{|c|c|c|c|c|c|}
\hline Factors & Baseline & 6th Month & Mean Differences & $95 \% \mathrm{Cl}$ & p value \\
\hline \multicolumn{6}{|l|}{ Dose } \\
\hline \multicolumn{6}{|c|}{$10-20 \mathrm{mg}(\mathrm{n}=210)$} \\
\hline Chol & I69.7I (45.68) & 163.64 (35.88) & -6.07 & $-12.34,0.19$ & 0.057 \\
\hline $\mathrm{Tg}$ & 134.33 (62.48) & 136.70 (6I.7I) & 2.37 & $-66.55,11.29$ & 0.600 \\
\hline HDL & $53.33(12.49)$ & $50.85(12.64)$ & -2.48 & $-3.64,-\mid .31$ & $<0.001$ \\
\hline LDL & $105.69(4 \mid .7 I)$ & $97.50(31.88)$ & -8.18 & $-13.20,-3.15$ & 0.001 \\
\hline \multicolumn{6}{|c|}{$40-80 \mathrm{mg}(\mathrm{n}=278)$} \\
\hline Chol & $178.14(52.25)$ & I68.1I (49.84) & -10.02 & $-15.45,-4.59$ & $<0.001$ \\
\hline $\mathrm{Tg}$ & I52.58 (74.42) & 153.08 (88.17) & 0.49 & $-9.69,10.68$ & 0.923 \\
\hline HDL & $50.24(14.04)$ & $48.40(13.30)$ & -1.83 & $-3.30,-0.37$ & 0.014 \\
\hline LDL & II 3.40 (46.67) & $103.26(49.74)$ & -10.14 & $-15.43,-4.84$ & $<0.001$ \\
\hline
\end{tabular}

Abbreviations: Cl, confidence interval; Chol, cholesterol (mg/dL); Tg, triglyceride (mg/dL); HDL, high-density lipoprotein-cholesterol (mg/dL); LDL, low-density lipoprotein-cholesterol (mg/dL).

Table 7 Lipid Parameters of Dyslipidemia Patients Before Switching Treatment from Brand-Name to Generic Atorvastatin and 6 Months After Stratified by Indications for Dyslipidemia Treatment

\begin{tabular}{|c|c|c|c|c|c|}
\hline Factors & Baseline & 6th Month & Mean Differences & $95 \% \mathrm{Cl}$ & p value \\
\hline \multicolumn{6}{|c|}{ Indications } \\
\hline \multicolumn{6}{|c|}{ Primary $(n=398)$} \\
\hline Chol & 176.08 (48.27) & $167.08(44.26)$ & -9.00 & $-13.53,-4.46$ & $<0.001$ \\
\hline $\mathrm{Tg}$ & I42.37 (68.8I) & I43.76 (78.56) & 1.38 & $-6.32,9.10$ & 0.723 \\
\hline HDL & $52.04(13.36)$ & $50.04(13.14)$ & -2.00 & $-3.04,-0.96$ & $<0.001$ \\
\hline LDL & III.6I (44.32) & 101.75 (44.48) & -9.85 & $-14.05,-5.64$ & $<0.001$ \\
\hline \multicolumn{6}{|c|}{ Secondary $(n=90)$} \\
\hline Chol & 166.15 (55.43) & $161.46(44.25)$ & -4.69 & $-|4.30,4.9|$ & 0.332 \\
\hline $\mathrm{Tg}$ & $153.93(74.10)$ & I55.| 4 (7I.40) & 1.20 & $-12.78,15.18$ & 0.864 \\
\hline HDL & $49.48(13.72)$ & $46.67(12.25)$ & -2.80 & $-5.28,-0.32)$ & 0.027 \\
\hline LDL & $103.33(46.11)$ & $96.48(35.81)$ & -6.84 & $-14.5 \mid, 0.83$ & 0.079 \\
\hline
\end{tabular}

Abbreviations: $\mathrm{Cl}$, confidence interval; Chol, cholesterol (mg/dL); Tg, triglyceride (mg/dL); HDL, high-density lipoprotein-cholesterol (mg/dL); LDL, low-density lipoprotein-cholesterol (mg/dL).

study, it is possible that there were confounding factors, and we were unable to evaluate any impact on long-term morbidity and morbidity.

We found that generic atorvastatin resulted in significantly lower LDL-c than brand-name atorvastatin but less of an increase in HDL-c. Further randomized controlled trials over longer study periods may be necessary.

\section{Disclosure}

The authors report no conflicts of interest in this work.

\section{References}

1. GBD 2013 Mortality and Causes of Death Collaborators. Global, regional, and national age-sex specific all-cause and cause-specific mortality for 240 causes of death, 1990-2013: a systematic analysis for the Global Burden of Disease Study 2013. Lancet. 2015;385 (9963):117-171. doi:10.1016/S0140-6736(14)61682-2 
2. Yusuf S, Hawken S, Ounpuu S, et al. Effect of potentially modifiable risk factors associated with myocardial infarction in 52 countries (the INTERHEART study): case-control study. Lancet. 2004;364 (9438):937-952. doi:10.1016/S0140-6736(04)17018-9

3. Roncaglioni MC, Santoro L, D'Avanzo B, et al. Role of family history in patients with myocardial infarction. An Italian case-control study. GISSI-EFRIM investigators. Circulation. 1992;85(6):2065-2072. doi:10.1161/01.CIR.85.6.2065

4. Genest JJ Jr, Martin-Munley SS, McNamara JR, et al. Familial lipoprotein disorders in patients with premature coronary artery disease. Circulation. 1992;85(6):2025-2033. doi:10.1161/01.CIR.85.6.2025

5. Das M, Choudhury S, Maity S, et al. Generic versus branded medicines: an observational study among patients with chronic diseases attending a public hospital outpatient department. J Nat Sci Biol Med. 2017;8(1):26-31. doi:10.4103/0976-9668.198351

6. Akunne OO, Godman B, Adedapo AD, Truter I, Fadare J. Statin prescribing among hypertensive patients in southwest Nigeria: findings and implications for the future. J Comp Eff Res. 2016;5 (3):281-288. doi:10.2217/cer.15.65

7. Kim SH, Seo MK, Yoon MH, Choi DH, Hong TJ, Kim HS. Assessment of the efficacy and tolerability of 2 formulations of atorvastatin in Korean adults with hypercholesterolemia: a multicenter, prospective, open-label, randomized trial. Clin Ther. 2013;35(1):77-86. doi:10.1016/j.clinthera.2012.11.009

8. Kim SH, Park K, Hong SJ, et al. Efficacy and tolerability of a generic and a branded formulation of atorvastatin $20 \mathrm{mg} / \mathrm{d}$ in hypercholesterolemic Korean adults at high risk for cardiovascular disease: a multicenter, prospective, randomized, double-blind, double-dummy clinical trial. Clin Ther. 2010;32(11):1896-1905. doi:10.1016/j. clinthera.2010.10.004

9. Boh M, Opolski G, Poredos P, Ceska R, Jezovnik M. Therapeutic equivalence of the generic and the reference atorvastatin in patients with increased coronary risk. Int Angiol. 2011;30(4):366-374.
10. Ong LM, Punithavathi N, Lena YL, Mahanim O, Leekha S; Storvas Clinical Trial Study Group. Long-term efficacy and safety of a generic atorvastatin in usual clinical care setting. Med J Malaysia. 2011;66(3):214-219.

11. Loch A, Bewersdorf JP, Kofink D, Ismail D, Abidin IZ, Veriah RS. Generic atorvastatin is as effective as the brand-name drug (LIPITOR $^{\circledR}$ ) in lowering cholesterol levels: a cross-sectional retrospective cohort study. BMC Res Notes. 2017;10(1):291. doi:10.1186/ s13104-017-2617-6

12. Wang KY, Ting CT. A randomized, double-blind, placebo-controlled, 8-week study to evaluate the efficacy and safety of once daily atorvastatin $(10 \mathrm{mg})$ in patients with elevated LDL-cholesterol. Jpn Heart J. 2001;42(6):725-738. doi:10.1536/jhj.42.725

13. Rahalkar AR, Ban MR, Hegele RA. Clinical equivalence of proprietary and generic atorvastatin in lipid clinic patients. Can J Cardiol. 2013;29(4):418-422. doi:10.1016/j.cjca.2012.05.010

14. McTaggart F, Jones P. Effects of statins on high-density lipoproteins: a potential contribution to cardiovascular benefit. Cardiovasc Drugs Ther. 2008;22(4):321-338. doi:10.1007/s10557-008-6113-Z

15. Clarke AT, Johnson PC, Hall GC, Ford I, Mills PR. High dose atorvastatin associated with increased risk of significant hepatotoxicity in comparison to simvastatin in UK GPRD cohort. PLoS One. 2016;11(3):e0151587. doi:10.1371/journal.pone.0151587

16. Sawunyavisuth B. What are predictors for a continuous positive airway pressure machine purchasing in obstructive sleep apnea patients? Asia Pac J Sci Technol. 2018;23:APST-23-03-10.

17. Kingkaew N, Antadech T. Cardiovascular risk factors and 10-year CV risk scores in adults aged 30-70 years old in Amnat Charoen Province, Thailand. Asia Pac J Sci Technol. 2019;24:APST-24-04-04.

18. Buttichak A, Leelayuwat N, Bumerraj S, Boonprakob Y. The effects of a yoga training program with fit ball on the physical fitness and body composition of overweight or obese women. Asia Pac J Sci Technol. 2019;24:APST-24-02-07.
Clinical Pharmacology: Advances and Applications is an international, peer-reviewed, open access journal publishing original research, reports, reviews and commentaries on all areas of drug experience in humans. The manuscript management system is completely online and includes a very quick and fair peer-review system, which is all easy to use. Visit http://www.dovepress.com/testimonials.php to read real quotes from published authors. 